PROCEEDINGS OF THE

AMERICAN MATHEMATICAL SOCIETY

Volume 131, Number 9, Pages 2673-2679

S 0002-9939(03)06815-1

Article electronically published on February 20, 2003

\title{
AN AFFINE PI HOPF ALGEBRA NOT FINITE OVER A NORMAL COMMUTATIVE HOPF SUBALGEBRA
}

\author{
SHLOMO GELAKI AND EDWARD S. LETZTER
}

(Communicated by Martin Lorenz)

\begin{abstract}
In formulating a generalized framework to study certain noncommutative algebras naturally arising in representation theory, K. A. Brown asked if every finitely generated Hopf algebra satisfying a polynomial identity was finite over a normal commutative Hopf subalgebra. In this note we show that Radford's biproduct, applied to the enveloping algebra of the Lie superalgebra $\mathrm{pl}(1,1)$, provides a noetherian prime counterexample.
\end{abstract}

\section{INTRODUCTION}

Noncommutative affine PI Hopf algebras arise in fundamental representationtheoretic contexts: as enveloping algebras of finite dimensional restricted Lie algebras in positive characteristic, as group algebras of finitely generated abelian-byfinite groups, and as quantizations of algebraic groups - and their corresponding Lie algebras - at roots of unity. (By affine we mean "finitely generated over the ground field as an associative algebra," and by PI we mean "satisfying a polynomial identity.") In all of the preceding examples, the Hopf algebra of interest is finitely generated as a module over a normal affine commutative Hopf subalgebra. Therefore, in developing a general structure theory to handle these examples, K. A. Brown asked [3, Question B] whether every affine PI Hopf algebra was finitely generated as a left (or right) module over some normal commutative Hopf subalgebra (cf. [4]). In this note we present a counterexample; we describe a prime affine noetherian PI Hopf algebra not finitely generated as a left or right module over any of its normal commutative Hopf subalgebras. This counterexample also arises in a representation-theoretic context, resulting from an application of Radford's biproduct [12] to the enveloping algebra of the Lie superalgebra $\mathrm{pl}(1,1)$. (In the terminology of [10, the counterexample is the "bosonization" of the enveloping algebra of $\mathrm{pl}(1,1)$.)

Received by the editors December 5, 2001 and, in revised form, April 5, 2002

2000 Mathematics Subject Classification. Primary 16W30; Secondary 16R20, 16W55.

The first author's research was supported by the Technion V.P.R. Fund-Loewengart Research Fund, and by the Fund for the Promotion of Research at the Technion. The second author's research was supported in part by NSF grant DMS-9970413. This research was begun during the second author's visit to the Technion in August 2001, and he is grateful to the Technion for its hospitality. 


\section{Preparatory Results}

Let $k$ be an algebraically closed field of characteristic zero. We assume throughout this note that $k$ is the ground field for all of the vector spaces mentioned (explicitly or implicitly). In particular, all algebras (associative and otherwise) are over $k$. All of the associative algebras discussed below will be assumed to be unital.

The reader is referred to 99 for background on noncommutative rings, to 11 for background on Hopf algebras, and to [13] for background on Lie superalgebras.

2.1. (i) For all of the Hopf algebras $H$ we will encounter, we will use $\Delta_{H}$ to denote the coproduct, $\epsilon_{H}$ to denote the counit, and $S_{H}$ to denote the antipode. The subscripts will be dropped when the meaning is clear.

(ii) Let $H$ be a Hopf algebra. Recall the left and right adjoint actions of $H$ on itself,

$$
\left(\operatorname{ad}_{l} a\right)(b)=\sum a_{1} b S\left(a_{2}\right) \quad \text { and } \quad\left(\operatorname{ad}_{r} a\right)(b)=\sum S\left(a_{1}\right) b a_{2}
$$

for $a, b \in H$, where $\Delta(a)=\sum a_{1} \otimes a_{2}$. Also recall that a Hopf subalgebra $A$ of $H$ is normal in $H$ provided $\left(\operatorname{ad}_{l} H\right)(A)$ and $\left(\operatorname{ad}_{r} H\right)(A)$ are contained in $A$.

2.2. Next we briefly review some superalgebra.

(i) A vector space $V$ equipped with a $\mathbb{Z}_{2}$-grading is termed a supervector space; we indicate the grading by writing $V=V_{0} \oplus V_{1}$. The vectors in $V_{0}$ are called even and those in $V_{1}$ are called odd. We define the parity of a homogeneous vector $v \in V$ to be $p(v)=0$ if $v$ is even and $p(v)=1$ if $v$ is odd.

We will use SuperVect to denote the category of supervector spaces with homogeneous morphisms. For $V, W \in$ SuperVect the commutativity constraint $V \otimes W \rightarrow$ $W \otimes V$ is different from the classical one and is given by the formula

$$
v \otimes w \mapsto(-1)^{p(v) p(w)} w \otimes v,
$$

for homogeneous $v \in V$ and $w \in W$.

(ii) One can define Lie algebras, bialgebras, Hopf algebras, etc. in SuperVect by replacing the ordinary structure maps with morphisms in SuperVect. The resulting objects are referred to, respectively, as Lie superalgebras, superbialgebras, Hopf superalgebras, etc.

For example, a Hopf superalgebra $H$ is a $\mathbb{Z}_{2}$-graded associative algebra, possessing a coassociative multiplicative (in the super-sense) morphism $\Delta: H \rightarrow H \otimes H$ in SuperVect, and further equipped with a counit $\epsilon$ and antipode $S$ satisfying the expected axioms. By "multiplicativity in the super-sense" we mean that $\Delta$ satisfies the relation

$$
\Delta(a b)=\sum(-1)^{p\left(a_{2}\right) p\left(b_{1}\right)} a_{1} b_{1} \otimes a_{2} b_{2}
$$

for all homogeneous $a, b \in H$ (where $\Delta(a)=\sum a_{1} \otimes a_{2}, \Delta(b)=\sum b_{1} \otimes b_{2}$ and the components are homogeneous). This formulation is required because the tensor product of algebras $A$ and $B$ in SuperVect is defined to be the vector space $A \otimes B$ equipped with the multiplication

$$
(a \otimes b)\left(a^{\prime} \otimes b^{\prime}\right):=(-1)^{p\left(a^{\prime}\right) p(b)} a a^{\prime} \otimes b b^{\prime},
$$

for homogeneous $a, a^{\prime} \in A$ and $b, b^{\prime} \in B$. (Hopf superalgebras are referred to as "graded Hopf algebras" in [7].)

(iii) Henceforth, "graded" will mean " $\mathbb{Z}_{2}$-graded." 
2.3. (i) The Hopf superalgebras we are most concerned with in this note arise as follows: Let $\mathfrak{g}$ be a Lie superalgebra, and let $U(\mathfrak{g})$ be the enveloping algebra of $\mathfrak{g}$. Then $U(\mathfrak{g})$ is a Hopf superalgebra with coproduct $\Delta$, counit $\epsilon$, and antipode $S$ determined by

$$
\Delta(x)=x \otimes 1+1 \otimes x, \quad S(x)=-x, \quad \epsilon(x)=0,
$$

for all $x \in \mathfrak{g}$. Note that $U(\mathfrak{g})$ is supercocommutative with no nontrivial grouplike elements.

(ii) Now suppose that $U^{\prime}$ is a sub-Hopf superalgebra of $U(\mathfrak{g})$. By a theorem of Kostant 7, Theorem 3] (cf. 11), there exists a sub-Lie superalgebra $\mathfrak{a}$ of $\mathfrak{g}$ such that $U^{\prime}=U(\mathfrak{a}) \subseteq U$. (We can apply Kostant's theorem because $U$, and hence also $U^{\prime}$, is supercocommutative with no nontrivial grouplike elements.) Note that $\mathfrak{a}_{0}$ is contained in $\mathfrak{g}_{0}$ and that $\mathfrak{a}_{1}$ is contained in $\mathfrak{g}_{1}$.

2.4. Let $U$ be any Hopf superalgebra.

(i) Recall (see, e.g., [1]) that Radford's biproduct [12] allows us to associate to $U$ a Hopf algebra $\bar{U}$ in the following way. As an algebra,

$$
\bar{U}=U \# k[\langle t\rangle] \cong U \# k[\mathbb{Z} / 2 \mathbb{Z}]
$$

is the smash product of $U$ and $k[\langle t\rangle]$, where $t$ is the graded algebra automorphism of $U$ (of order 2) acting on $U$ by parity (i.e., tat $=(-1)^{p(a)} a$ for all homogeneous $a \in U)$. The coproduct $\Delta$, counit $\epsilon$, and antipode $S$ of $\bar{U}$ are determined by

$$
\Delta(x)=\sum x_{1} \# t^{p\left(x_{2}\right)} \otimes x_{2} \# 1, \quad \Delta(t)=t \otimes t,
$$

and

$$
S(x)=t^{p(x)} S_{U}(x), \quad \epsilon(x)=\epsilon_{U}(x), \quad S(t)=t, \quad \epsilon(t)=1,
$$

where $x \in U$ is homogeneous and $\Delta_{U}(x)=\sum x_{1} \otimes x_{2}$ with homogeneous components.

(ii) In particular, $K:=k[\langle t\rangle]$ is a Hopf subalgebra of $\bar{U}$, and there is a unique Hopf algebra projection

$$
\bar{U} \stackrel{\pi}{\rightarrow} K,
$$

sending $u \# h$ to $\epsilon(u) h$. Observe that

$$
U=\{x \in \bar{U}:(\mathrm{id} \otimes \pi) \Delta(x)=x \otimes 1\},
$$

where id denotes the identity map, is the algebra of $K$-coinvariants of $\bar{U}$ (see [12]).

(iii) Also note that $U$ is a subalgebra of $\bar{U}$, and there is a unique coalgebra projection

$$
\bar{U} \stackrel{\Pi}{\longrightarrow} U
$$

sending $u \# h$ to $u \epsilon(h)$.

(iv) Lastly, by declaring $t$ to be even, we can extend the grading on $U$ to a grading on $\bar{U}$. Note that $t a=(-1)^{p(a)} a t$ for all homogeneous $a \in \bar{U}$.

2.5. Let $\mathfrak{g}$ be a Lie superalgebra with enveloping algebra $U:=U(\mathfrak{g})$.

(i) The Hopf algebra structure on $\bar{U}$ follows from

$\Delta(x)=x \otimes 1+t^{p(x)} \otimes x, S(x)=-t^{p(x)} x, \epsilon(x)=0, \Delta(t)=t \otimes t, S(t)=t, \epsilon(t)=1$, for homogeneous $x \in \mathfrak{g}$ (see, e.g., [5]). 
(ii) Now choose homogeneous $a, b \in \mathfrak{g}$. Write $\Delta_{U}(a)=\sum a_{1} \otimes a_{2}$. Then

$$
\begin{aligned}
\left(\operatorname{ad}_{\mathfrak{g}} a\right)(b) & =[a, b]=a b-(-1)^{p(b) p(a)} b a=\sum a_{1} b(-1)^{p(b) p\left(a_{2}\right)} S_{U}\left(a_{2}\right) \\
& =\sum\left(a_{1} \# 1\right)(b \# 1)(-1)^{p(b) p\left(a_{2}\right)}\left(1 \# t^{p\left(a_{2}\right)}\right) S\left(a_{2} \# 1\right) \\
& =\sum\left(a_{1} \# t^{p\left(a_{2}\right)}\right)(b \# 1) S\left(a_{2} \# 1\right)=\left(\operatorname{ad}_{l} a\right)(b),
\end{aligned}
$$

where $\operatorname{ad}_{l}$ denotes the left adjoint action of $\bar{U}$ on itself, and $\operatorname{ad}_{\mathfrak{g}}$ denotes the adjoint (graded) representation of $\mathfrak{g}$ in itself. Consequently, if $A$ is a normal Hopf subalgebra of $\bar{U}$ such that $A \cap U=U(\mathfrak{a})$, for some Lie superalgebra $\mathfrak{a}$ of $\mathfrak{g}$, then $\mathfrak{a}$ is an ideal of $\mathfrak{g}$. of $\bar{U}$.

(iii) Note that $U\left(\mathfrak{g}_{0}\right)$ is both a sub-Hopf superalgebra of $U$ and a Hopf subalgebra

2.6. Lemma. Let $U$ be any Hopf superalgebra, and let $A$ be a Hopf subalgebra of $\bar{U}$ containing t. Then

(i) $A \cap U$ is a sub-Hopf superalgebra of $U$. In particular, if $U$ is the enveloping algebra of a Lie superalgebra $\mathfrak{g}$, then $A \cap U=U(\mathfrak{a})$ for some sub-Lie superalgebra $\mathfrak{a}$ of $\mathfrak{g}$.

(ii) $A=(A \cap U) \# K$.

Proof. Set $\Delta:=\Delta_{\bar{U}}$ and $S:=S_{\bar{U}}$. To begin, $\pi(A)=K$ since $t \in A$. Also, the composition of Hopf algebra homomorphisms

$$
K \stackrel{i}{\rightarrow} A \stackrel{i}{\rightarrow} \bar{U} \stackrel{\pi}{\rightarrow} K
$$

where $i$ denotes the inclusion map, reduces to the identity map on $K$. Hence, by Theorem 3 in [12], we obtain a biproduct decomposition

$$
A=\tilde{A} \# K,
$$

where, by construction, $\tilde{A}=\Pi(A)$ is a Hopf superalgebra. Therefore, it is straightforward to verify that

$$
\tilde{A}=\{x \in A:(\operatorname{id} \otimes \pi) \Delta(x)=x \otimes 1\}=A \cap U,
$$

is a subalgebra of $U$. Moreover, since $A$ and $U$ are stable under the (left or, equivalently, right) adjoint action of $t$, we see that $A \cap U$ is stable under the (left or, equivalently right) adjoint action of $t$. Therefore, $A \cap U$ is a graded subalgebra of $U$. Also, since $\Pi$ is a coalgebra map, we can conclude that $A \cap U=\tilde{A}=\Pi(A)$ is a subcoalgebra of $U$.

Finally, if $a$ is a homogeneous element of $A \cap U$, then $S(a)=t^{p(a)} S_{U}(a) \in A$. Hence $S_{U}(a)=t^{p(a)} S(a) \in A$, and we see that $S_{U}(A \cap U) \subseteq A \cap U$. In view of (2.3ii), the lemma now follows.

\section{The EXAmple}

3.1. In this section we let $\mathfrak{g}=\mathfrak{g}_{0} \oplus \mathfrak{g}_{1}$ denote the Lie superalgebra $\mathrm{pl}(1,1)$ of $2 \times 2$ matrices. Fix a basis for $\mathfrak{g}$,

$$
x=\left[\begin{array}{ll}
1 & 0 \\
0 & 1
\end{array}\right], \quad y=\left[\begin{array}{ll}
1 & 0 \\
0 & 0
\end{array}\right], \quad u=\left[\begin{array}{ll}
0 & 1 \\
0 & 0
\end{array}\right], \quad v=\left[\begin{array}{ll}
0 & 0 \\
1 & 0
\end{array}\right] .
$$


Recall that $\mathfrak{g}_{0}$ is generated by $x$ and $y$, while $\mathfrak{g}_{1}$ is generated by $u$ and $v$. Set $U:=U(\mathfrak{g})$. As in (2.4i), let $\bar{U}:=U \# k[\langle t\rangle]$. The relations defining $\bar{U}$ as an algebra are

$$
\begin{aligned}
x h=h x \quad \text { for all } h \in \bar{U}, \quad y u-u y=u, \quad y v-v y=-v, \quad u v+v u=x, \\
u^{2}=v^{2}=0, \quad t x-x t=t y-y t=0, \quad t u=-u t, \quad t v=-v t, \quad t^{2}=1 .
\end{aligned}
$$

We can now state the main result of this paper.

3.2. Theorem. The Hopf algebra $\bar{U}$ is a noetherian prime affine PI Hopf algebra not finitely generated, as a right or left module, over any of its normal commutative Hopf subalgebras.

The remainder of this section is devoted to a proof of the theorem

3.3. (i) Note that $u$ is an $\operatorname{ad}_{\mathfrak{g}}(y)$-eigenvector with eigenvalue 1 and that $v$ is an $\operatorname{ad}_{\mathfrak{g}}(y)$-eigenvector with eigenvalue -1 .

(ii) Let $w$ be an $\operatorname{ad}_{\mathfrak{g}}(y)$-eigenvector of $\mathfrak{g}_{1}$. By (i), the corresponding eigenvalue is \pm 1 . Hence, in $\bar{U}$, for all positive integers $n$, either $y^{n} w=w(y+1)^{n}$ or $y^{n} w=$ $w(y-1)^{n}$.

3.4. Let $F$ be an algebra containing a subalgebra $E$, and suppose that $F$ is finitely generated on the right (or left) as an $E$-module. Noting that $F$ is a ring subquotient of a matrix ring over $E$, we see that $E$ is PI if and only if $F$ is PI. Next, we will also require some basic and well-known properties of Gelfand-Kirillov dimension (abbreviated to "GK dimension" or "GKdim"); see [8] or 9. Chapter 8] for background. In particular, GKdim $E=\operatorname{GKdim} F$ (see, e.g., [8, 5.5]). The GK dimension of the commutative polynomial algebra $k\left[x_{1}, \ldots, x_{n}\right]$ is equal to $n$, and the enveloping algebra of an $n$-dimensional Lie algebra has GK dimension equal to $n$.

3.5. Note that $k[x, y]=k\langle x, y\rangle \subset \bar{U}$ is a commutative polynomial ring in $x$ and $y$. Moreover, $\bar{U}$ is finitely generated as a left and a right $k[x, y]$-module. Therefore, by (3.4), $\bar{U}$ is an affine noetherian PI algebra of GK dimension 2.

3.6. Suppose that $\mathfrak{a}$ is a sub-Lie superalgebra of $\mathfrak{g}$ such that $\mathfrak{a}_{0} \neq \mathfrak{g}_{0}$. Then $\operatorname{dim} \mathfrak{a}_{0} \leq$ 1 , and it follows that $\operatorname{GKdim} U\left(\mathfrak{a}_{0}\right) \leq 1$. It follows from the Poincaré-Birkhoff-Witt Theorem for Lie superalgebras (PBW Theorem) that $U(\mathfrak{a})$ is finitely generated as a left $U\left(\mathfrak{a}_{0}\right)$-module. Hence, by (3.4), we see that $\operatorname{GKdim} U(\mathfrak{a}) \leq 1$.

3.7. Let $C$ be a commutative normal Hopf subalgebra of $\bar{U}$. We now prove that $\bar{U}$ cannot be finitely generated as a left or right module over $C$.

Case 1. Assume that $t \notin C$.

Set $A:=C\langle t\rangle$, and observe that $A$ is a Hopf subalgebra of $\bar{U}$. Since $C$ is stable under the left and right adjoint actions of $t$, we see that $C$ is a graded associative subalgebra of $\bar{U}$. Since $t$ is homogeneous, the grading on $C$ extends to a grading on $A$. For $a, b \in W:=C_{0} \cup C_{1} \cup\{t\}$, we further see that $a b= \pm b a$. Also, $A$ is generated as a $k$-algebra by $W$.

Now choose $a_{1}, \ldots, a_{n} \in W$. Set $A^{\prime}:=k\left\langle a_{1}, \ldots, a_{n}\right\rangle$, and set $Z^{\prime}:=k\left\langle a_{1}^{2}, \ldots, a_{n}^{2}\right\rangle$. Then $Z^{\prime}$ is central in $A$, and $A^{\prime}$ is generated as a left (or right) $Z^{\prime}$-module by the products $a_{1}^{i_{1}} \cdots a_{n}^{i_{n}}$, for $i_{1}, \ldots, i_{n} \in\{0,1\}$. In particular, $A^{\prime}$ is finitely generated as 
a left (or right) module over the commutative noetherian $\operatorname{ring} Z^{\prime}$. It follows that $A$ is integral over its center, in the sense of [9, 5.3.2].

Since $t \in A$, it follows from (2.6i) that $A \cap U=U(\mathfrak{a})$ for some sub-Lie superalgebra $\mathfrak{a}$ of $\mathfrak{g}$.

Subcase 1a. Assume that $\mathfrak{a}_{0}=\mathfrak{g}_{0}$ (and so $y \in A$ ).

First suppose that $\mathfrak{a}_{1} \neq 0$, ensuring that $\mathfrak{a}_{1}$ contains an $\operatorname{ad}_{\mathfrak{g}}(y)$-eigenvector. We can impose a nonnegative $\mathbb{Z}$-grading on $\mathfrak{g}$ by setting $\operatorname{deg}(y)=0, \operatorname{deg}(u)=\operatorname{deg}(v)=$ 1 , and $\operatorname{deg}(x)=2$. This $\mathbb{Z}$-grading restricts to $\mathfrak{a}$ since $\mathfrak{a}$ must be generated by $\mathbb{Z}$ homogeneous elements. Moreover, by declaring $\operatorname{deg}(t)=0$, we obtain a $\mathbb{Z}$-grading on $A$. Note that the 0 -degree $\mathbb{Z}$-homogeneous component of $A$ is $k[y, t]$, and also note that the $\mathbb{Z}$-grading on $A$ restricts to the center of $A$. It now follows from the PBW Theorem, and (3.3ii), that the 0-degree $\mathbb{Z}$-homogeneous component of the center is $k$. However, we learned above that $y$ must be integral over the center of $A$, and so $y$ must be integral over the 0 -degree $\mathbb{Z}$-homogeneous component of the center of $A$. Because $y$ cannot be integral over $k$, we have arrived at a contradiction. Therefore, $\mathfrak{a}_{1}=0$.

It now follows that $A \cap U=U\left(\mathfrak{g}_{0}\right)$, and it follows from (2.6ii) that $A=U\left(\mathfrak{g}_{0}\right) \# K$. In particular, $A$ is a pointed cocommutative Hopf algebra, and the set of grouplike elements of $A$ is $\{1, t\}$. Also, $\mathfrak{g}_{0}$ is the Lie algebra of primitive elements of $A$. Now, since $t \notin C$, we see that $C$ is a cocommutative connected Hopf algebra, and again by a theorem of Kostant (see, e.g., [11, 5.6.5]), we find that $C=U(\mathfrak{c})$, where $\mathfrak{c}$ is the Lie algebra of primitive elements of $C$. Of course, $\mathfrak{c} \subseteq \mathfrak{g}_{0}$, and so $\mathfrak{c}=\mathfrak{c}_{0}$ is an even sub-Lie superalgebra of $\mathfrak{g}$. Moreover, it follows from (2.5ii) that $\mathfrak{c}$ is an ideal of $\mathfrak{g}$. Now, any ideal of $\mathfrak{g}$ containing $y$ must also contain $u$ and $v$, and so we see that $y \notin \mathfrak{c}$. Thus $\mathfrak{c} \neq \mathfrak{g}_{0}$. Therefore, $\operatorname{GKdim} C \leq 1$, by (3.6), and so $\bar{U}$ cannot be a finitely generated left or right $C$-module, since $G K \operatorname{dim} \bar{U}=2$.

Subcase 1b. Assume that $\mathfrak{a}_{0} \neq \mathfrak{g}_{0}$.

It now follows from (3.6) that GKdim $A \cap U \leq 1$. By (2.6ii), $A$ is finitely generated as a left $A \cap U$-module, and so by (3.4), GKdim $A \leq 1$. Therefore, $\bar{U}$ cannot be finitely generated as a left or right $A$-module. Hence $\overline{\bar{U}}$ cannot be finitely generated as a left or right $C$-module.

Case 2. Assume that $t \in C$.

By (2.6i), it follows that $C \cap U=U(\mathfrak{c})$, for some sub-Lie superalgebra $\mathfrak{c}$ of $U$. Moreover, it follows from (2.5ii) that $\mathfrak{c}$ is an ideal of $\mathfrak{g}$. Consequently, if $\mathfrak{c}_{0}=\mathfrak{g}_{0}$, then $\mathfrak{c}=\mathfrak{g}$. But $\mathfrak{c} \neq \mathfrak{g}$, since otherwise $U$ is equal to the commutative algebra $C \cap U$. So $\mathfrak{c}_{0} \neq \mathfrak{g}_{0}$, and we deduce from (3.6) that GKdim $C \cap U \leq 1$. Hence, by (2.6ii) and (3.4), GKdim $C \leq 1$. We conclude that $\bar{U}$ cannot be a finitely generated left or right $C$-module.

3.8. It only remains to prove that $\bar{U}$ is prime. Let $R$ be a prime noetherian ring, and let $\tau$ be an automorphism of $R$ of order 2. Let $T$ denote the Ore extension $R[Y ; \tau]$. It follows, for example, from [6, 2.3i-iii] that $T /\left\langle Y^{2}-1\right\rangle$ is prime. As noted in [2], $U$ is prime. Therefore, since $\bar{U}$ is isomorphic as a ring to $U[Y ; t] /\left\langle Y^{2}-1\right\rangle$, we see that $\bar{U}$ is prime.

3.9. Remark. Let $\mathfrak{b}$ be the sub-Lie superalgebra of $\mathfrak{g}$ generated by $y$ and $u$. Using the methods of this note, one can show that $U(\mathfrak{b}) \# k[\langle t\rangle]$ is a noetherian Hopf PI algebra, of GK dimension 1, not finitely generated as a right or left module over any 
of its normal commutative Hopf subalgebras. However, the ideal $\langle u\rangle$ of $U(\mathfrak{b}) \# k[\langle t\rangle]$ is nilpotent, and so $U(\mathfrak{b}) \# k[\langle t\rangle]$ is not semiprime. Now recall, as a consequence of the Small-Warfield Theorem [14], that a prime affine PI algebra of GK dimension 1 must be finite over its center. We therefore ask: Does there exist a prime affine PI Hopf algebra of GK dimension 1 that is not finitely generated as a left or right module over any of its normal commutative Hopf subalgebras?

\section{ACKNOWLEDGMENT}

We are grateful to K. A. Brown and Pavel Etingof for reading preliminary versions of this manuscript, and for numerous useful and stimulating conversations.

\section{REFERENCES}

1. N. Andruskiewitsch, P. Etingof, and S. Gelaki, Triangular Hopf algebras with the Chevalley property, Michigan Journal of Mathematics 49 (2001), 277-298. MR 2002h:16057

2. E. J. Behr, Enveloping algebras of Lie superalgebras, Pacific J. Math 130 (1987), 9-25. MR 89b: 17023

3. K. A. Brown, Representation theory of noetherian Hopf algebras satisfying a polynomial identity, Trends in the Representation Theory of Finite Dimensional Algebras, Contemporary Mathematics, vol. 229, American Mathematical Society, Providence, 1998, pp. 49-79. MR 99m:16056

4. K. A. Brown and K. R. Goodearl, Lectures on Algebraic Quantum Groups (to appear).

5. D. Fischman, Schur's double centralizer theorem: a Hopf algebra approach, J. Algebra 157 (1993), 331-340. MR 94e:16043

6. K. R. Goodearl and E. S. Letzter, Prime ideals in skew and q-skew polynomial rings, Mem. Amer. Math. Soc. 521 (1994). MR 94j:16051

7. B. Kostant, Graded manifolds, graded Lie theory, and prequantization, Differential Geometrical Methods in Mathematical Physics, Proc. Symp. Bonn 1975, Lect. Notes Math., vol. 570, Springer, New York, 1977, pp. 177-306. MR 58:28326

8. G. Krause and T. H. Lenagan, Growth of Algebras and Gelfand-Kirillov Dimension, revised edition, American Mathematical Society, Providence, 2000. MR 2000j:16035

9. J. C. McConnell and J. C. Robson, Noncommutative Noetherian Rings, Grad. Stud. Math., vol. 30, Amer. Math. Soc., Providence, 2000. MR 2001i:16039

10. S. Majid, Foundations of Quantum Group Theory, Cambridge University Press, Cambridge, 1995. MR 97g:17016

11. S. Montgomery, Hopf Algebras and Their Actions on Rings, Conference Board of the Mathematical Sciences Regional Conference Series in Mathematics, vol. 82, American Mathematical Society, providence, 1993. MR 94i:16019

12. D. Radford, The structure of Hopf algebras with a projection, J. Algebra 92 (1985), 322-347. MR 86k:16004

13. M. Scheunert, The Theory of Lie Superalgebras, Lect. Notes Math., vol. 716, Springer, New York, 1979, pp. 177-306. MR 80i:17005

14. L. W. Small and R. B. Warfield, Jr., Prime affine algebras of Gel'fand-Kirillov dimension one, J. Algebra 91 (1984), 386-389. MR 86h:16006 ISRAEL

Department of Mathematics, Technion-Israel Institute of Technology, Haifa 32000 ,

E-mail address: gelaki@math.technion.ac.il

Department of Mathematics, Temple University, Philadelphia, Pennsylvania 19122

E-mail address: letzter@math.temple.edu 